\title{
Col Thalamic deep brain stimulation for acquired dystonia in children and young adults: a phase 1 clinical trial
}

\author{
*Marta San Luciano, MD, MS, ${ }^{1}$ Amy Robichaux-Viehoever, MD, PhD, ${ }^{2}$ Kristen A. Dodenhoff, BA, ${ }^{1}$ \\ Melissa L. Gittings, BA, ${ }^{1}$ Aaron C. Viser, BA, ${ }^{1}$ Caroline A. Racine, PhD, ${ }^{3}$ Ian O. Bledsoe, MD, MS, ${ }^{1}$ \\ Christa Watson Pereira, PsyD, ${ }^{1}$ Sarah S. Wang, PhD, ${ }^{1}$ Philip A. Starr, MD, PhD, ${ }^{3}$ and \\ Jill L. Ostrem, MD'
}

'Department of Neurology, Weill Institute for Neurosciences, and ' 2 Department of Neurological Surgery, University of California, San Francisco, California; and 'Department of Neurology, Division of Child Neurology, Washington University in St. Louis, Missouri

\begin{abstract}
OBJECTIVE The aim of this study was to evaluate the feasibility and preliminary efficacy and safety of combined bilateral ventralis oralis posterior/ventralis intermedius (Vop/Vim) deep brain stimulation (DBS) for the treatment of acquired dystonia in children and young adults. Pallidal DBS is efficacious for severe, medication-refractory isolated dystonia, providing 50\%-60\% long-term improvement. Unfortunately, pallidal stimulation response rates in acquired dystonia are modest and unpredictable, with frequent nonresponders. Acquired dystonia, most commonly caused by cerebral palsy, is more common than isolated dystonia in pediatric populations and is more recalcitrant to standard treatments. Given the limitations of pallidal DBS in acquired dystonia, there is a need to explore alternative brain targets. Preliminary evidence has suggested that thalamic stimulation may be efficacious for acquired dystonia.
\end{abstract}

METHODS Four participants, 3 with perinatal brain injuries and 1 with postencephalitic symptomatic dystonia, underwent bilateral Vop/Vim DBS and bimonthly evaluations for 12 months. The primary efficacy outcome was the change in Burke-Fahn-Marsden Dystonia Rating Scale (BFMDRS) and Barry-Albright Dystonia Scale (BADS) scores between the baseline and 12-month assessments. Video documentation was used for blinded ratings. Secondary outcomes included evaluation of spasticity (Modified Ashworth Scale score), quality of life (Pediatric Quality of Life Inventory [PedsQL] and modified Unified Parkinson's Disease Rating Scale Part II [UPDRS-II] scores), and neuropsychological assessments. Adverse events were monitored for safety.

RESULTS All participants tolerated the procedure well, and there were no safety concerns or serious adverse events. There was an average improvement of $21.5 \%$ in the BFMDRS motor subscale score, but the improvement was only $1.6 \%$ according to the BADS score. Following blinded video review, dystonia severity ratings were even more modest. Secondary outcomes, however, were more encouraging, with the BFMDRS disability subscale score improving by $15.7 \%$, the PedsQL total score by $27 \%$, and the modified UPDRS-II score by $19.3 \%$. Neuropsychological assessment findings were unchanged 1 year after surgery.

CONCLUSIONS Bilateral thalamic neuromodulation by DBS for severe, medication-refractory acquired dystonia was well tolerated. Primary and secondary outcomes showed highly variable treatment effect sizes comparable to those of pallidal stimulation in this population. As previously described, improvements in quality of life and disability were not reflected in dystonia severity scales, suggesting a need for the development of scales specifically for acquired dystonia.

Clinical trial registration no.: NCT03078816 (clinicaltrials.gov)

https://thejns.org/doi/abs/10.3171/2020.7.PEDS20348

KEYWORDS dystonia; thalamic; deep brain stimulation; DBS; pediatric; functional neurosurgery

ABBREVIATIONS AC-PC = anterior commissure-posterior commissure; BADS = Barry-Albright Dystonia Scale; BFMDRS = Burke-Fahn-Marsden Dystonia Rating Scale; DBS = deep brain stimulation; GPi = globus pallidus pars interna; PedsQL = Pediatric Quality of Life Inventory; UCSF = University of California, San Francisco; UPDRS-II = Unified Parkinson's Disease Rating Scale Part II; Vim = ventralis intermedius; Vop = ventralis oralis posterior.

SUBMITTED May 5, 2020. ACCEPTED July 7, 2020.

INCLUDE WHEN CITING Published online November 27, 2020; DOI: 10.3171/2020.7.PEDS20348.

${ }^{*}$ M.S.L. and A.R.V. share first authorship of this work. 
$\mathrm{D}$ YSTONIA is a movement disorder seen in both children and adults and is characterized by sustained or intermittent muscle contractions causing abnormal, often repetitive, movements, postures, or both. ${ }^{1}$ Acquired dystonia, or dystonia resulting from damage to the nervous system or degenerative processes, especially secondary to perinatal brain injuries resulting in cerebral palsy, is far more common in pediatric populations than isolated dystonia and notoriously more recalcitrant to standard pharmacological and surgical treatments. ${ }^{2,3}$

Pallidal deep brain stimulation (DBS) is highly efficacious for severe, medication-refractory, generalized, segmental, and some focal isolated dystonia forms, ${ }^{4}$ providing long-term symptom control and improved function, with average improvements ranging from $50 \%$ to $60 \%$, depending on various factors. ${ }^{5}$ Unfortunately, the response rates of pallidal stimulation in acquired dystonia are only modest and unpredictable, with a mean reduction of dystonia symptoms of only approximately $23 \%$ and frequent nonresponders. ${ }^{6}$ Given the limitations of pallidal DBS in acquired dystonia, alternative brain targets are being explored, and the thalamus may be an attractive candidate.

Historically, patients with acquired dystonia have shown improvement after thalamotomy, although the procedure was eventually abandoned due to a tendency for loss of efficacy over time ${ }^{8}$ and a perceived high prevalence of side effects and complications, especially when performed bilaterally. ${ }^{9}$ Thalamic DBS has been evaluated for dystonia in a few small open-label studies, mostly in conjunction with pallidal stimulation. ${ }^{10-12}$ By targeting the border of the ventralis oralis posterior (Vop) and ventralis intermedius (Vim) nuclei, the stimulation might strategically affect inputs from both the globus pallidus pars interna (GPi) and the cerebellum, leading to a more effective treatment than pallidal stimulation alone, especially since acquired dystonia often is multifactorial and cerebellar pathways may be involved in its pathogenesis. ${ }^{13}$

In this study, we carefully explored the effect of thalamic Vop/Vim stimulation in a group of patients with acquired dystonia caused mainly by perinatal brain injuries, with the primary aim of evaluating its feasibility and safety, as well as taking a preliminary look at its efficacy, and setting the stage for future, larger-scale clinical trials.

\section{Methods}

This was a single-institution, open-label, phase 1 clinical trial of Vop/Vim DBS in children and young adults with severe acquired dystonia despite appropriate conventional treatment (clinical trial registration no. NCT03078816, clinicaltrials.gov). The study was approved by the institutional review board at the University of California, San Francisco (UCSF) and the Food and Drug Administration under an investigational device exemption (IDE G162033).

\section{Study Participants}

Participants were recruited from the UCSF Movement Disorders and Neuromodulation Center and the Pediatric Neurology Clinics at UCSF Benioff Children's Hospitals in San Francisco and Oakland, California. Participants were eligible if they were between 7 and 25 years of age by the day of surgery and had confirmed acquired dystonia diagnoses (for example, a clear history of hypoxicischemic brain injury preceding dystonic symptoms) with or without MRI abnormalities. To qualify, a patient's dystonia had to have been present for at least 6 months and severe enough to warrant surgical intervention. Inclusion in the study also required verification of relatively intact thalamic anatomy for 2 years prior to surgery. Exclusion criteria included pregnancy and/or breastfeeding; major comorbidities that would increase surgical risk; active infection at the time of surgery; requirement of diathermy, electroconvulsive therapy, or transcranial magnetic stimulation; presence of previously implanted neurostimulators, pacemakers, defibrillators, or metallic head implants; history of exposure to neuroleptic agents; dystonia caused by known genetic mutations; severe cognitive impairment or dementia (estimated nonverbal IQ < 70); and uncontrolled depression. For 1 participant, written informed consent and assent were obtained with help from a medical Spanish interpreter. For the 3 participants who completed the study and were younger than 18 years, written or verbal assent was also obtained. A data and safety monitoring board, which included a movement disorders neurologist and a neurosurgeon at UCSF who were not otherwise involved in the study, was arranged, meeting biannually and ad hoc to review trial progress. After completion of endpoint evaluation and prior to study separation, all participants with implants were provided ample opportunity to discuss the pros and cons of continuing with stimulation, stopping stimulation but leaving the DBS system in place, or stopping stimulation and removing part or all of the DBS system. Each of the 4 included participants elected to continue DBS stimulation, and 2 chose to continue being followed up clinically at our center. Figure 1 illustrates the flow of events for all participants.

\section{Outcome Assessments}

Baseline assessments were performed 60 days prior to DBS surgery, and final endpoint assessments were performed following 12 months of continuous thalamic stimulation. The Burke-Fahn-Marsden Dystonia Rating Scale (BFMDRS) and Barry-Albright Dystonia Scale (BADS) were administered by the treating movement disorder neurologists (A.R.V. and M.S.L.) before surgery and repeated bimonthly over the course of the study. These severity ratings were tested for reliability by a blinded investigator (I.O.B.), who independently repeated these assessments by viewing video recordings without knowledge of prior ratings, DBS status, or time point in the study. Spasticity was evaluated as well, according to bimonthly administrations of the Modified Ashworth Scale. The Pediatric Quality of Life Inventory (PedsQL) and a modified version of the Unified Parkinson's Disease Rating Scale Part II (UPDRS-II; previously used and modified for use in pantothenate kinase-associated neurodegeneration) ${ }^{14}$ were administered to the participants and caregivers at 6 and 12 months to assess the effect of DBS on quality of life.

Additionally, each participant underwent baseline and postoperative neuropsychological evaluations conducted by trained neuropsychologists (C.A.R. and C.W.P.). The battery included measures of intellectual abilities (crys- 


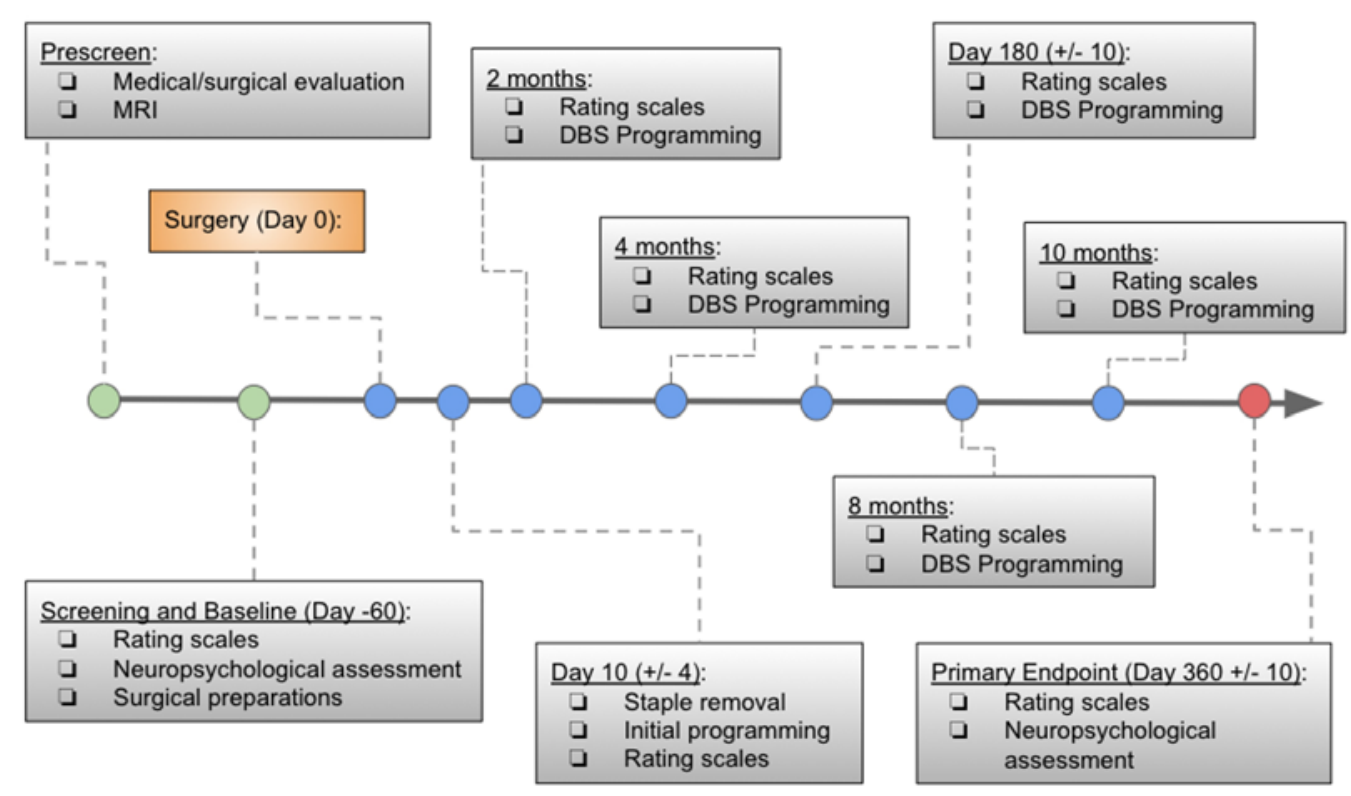

FIG. 1. Participant flowchart. Figure is available in color online only.

tallized and fluid tests of the Kaufman Brief Intelligence Test, 2nd Edition, ${ }^{15}$ Crystallized Scale and Fluid Scale), behaviors and emotion (Behavior Assessment System for Children, 3rd Edition, ${ }^{16}$ Self-Report of Personality for adolescents and Parent Rating Scale for adolescents [parent rating]), psychiatric symptoms (Diagnostic and Statistical Manual of Mental Disorders, 5th Edition, Mood Screener, ${ }^{17}$ self- and parent-rated), and speech (diadochokinetic syllables). Standardized normative data were used to transform raw scores into standard scores. All patients had difficulties with expressive language, and most relied on eye gaze communication, head nods, or other gestures to communicate responses to questions. Given the participants' differing visual and/or motor communication and fine motor control impairments, the psychometric testing was customized for each participant. Not all individuals were able to complete all measures due to motor difficulties, language impairment, and fatigue resulting from the effort and time required to communicate their answers.

\section{DBS Surgery}

Participants underwent implantation of bilateral DBS leads (3389 leads, Medtronic) in the Vop and Vim nuclei of the thalamus under general anesthesia with the aid of interventional MRI surgical guidance as described in previous publications from our center. ${ }^{18}$ Bilateral Medtronic Activa single-channel, nonrechargeable primary cell implantable pulse generators were implanted subcutaneously below the clavicle. The leads were implanted using a novel skull-mounted aiming device in conjunction with dedicated software (ClearPoint system), used in a 3T diagnostic MRI scanner (60-cm bore diameter, Philips Intera) in a radiology suite, with the patient under general anesthesia and without neurophysiological testing. Details on this methodology have been previously published..$^{18}$ The Vop/ Vim border within the thalamus was targeted approxi- mately $10 \mathrm{~mm}$ lateral to the ipsilateral border of the third ventricle and $6 \mathrm{~mm}$ anterior to the posterior commissure at the level of the anterior commissure-posterior commissure (AC-PC) plane. While it is difficult to visualize nuclear boundaries within the thalamus, we utilized fast gray matter acquisition T1 inversion recovery (FGATIR) imaging, reformatted in the axial and coronal planes, to visualize the thalamocapsular border. We adjusted the lateral coordinate and the exact lead trajectory to ensure that the contact area would be located 2-3 mm medial to this border. Postoperative MRI was performed in all participants and transferred to Framelink (Medtronic) to document lead locations. The coronal and sagittal approach angles and lead entry and tip positions in AC-PC coordinates are listed in Table 1. T1-weighted MR images of lead locations in all participants are shown in Fig. 2. Following the intervention, participants were transferred to the pediatric intensive care unit for 1-2 nights and then to the floor unit for postoperative care. Given that pediatric patients and wheelchair-bound patients carry a high risk of infection, all participants received an enhanced postoperative antibiotic regimen of 48 hours of intravenous vancomycin and ceftriaxone, followed by 10 days of oral dicloxacillin. This antibiotic regimen has been associated with significantly reduced numbers of infections compared with historical studies in this population. ${ }^{19}$

\section{Stimulation Optimization}

Stimulation was initiated approximately 1 month after implantation. During the first programming session, each of the 4 contacts was activated in a monopolar mode to perform a monopolar survey, with a single contact assigned to be the cathode and the implantable pulse generator as the anode. The pulse width and frequency were fixed at values typical for dystonia (pulse width $60 \mu \mathrm{sec}$, frequency 130 $\mathrm{Hz}$ ), and the amplitude was increased by $0.5-$ to $1-\mathrm{V}$ incre- 
TABLE 1. DBS electrode coordinates and settings

\begin{tabular}{|c|c|c|c|c|c|c|c|c|}
\hline \multirow{2}{*}{$\begin{array}{l}\text { Participant } \\
\text { No. }\end{array}$} & \multirow[b]{2}{*}{ Side } & \multirow{2}{*}{$\begin{array}{c}\text { AC-PC } \\
\text { Distance }\end{array}$} & \multicolumn{3}{|c|}{ Tip Coordinate* $^{*}$} & \multicolumn{2}{|c|}{ Approach Angle } & \multirow{2}{*}{$\begin{array}{l}\text { Final DBS } \\
\text { Settings }\end{array}$} \\
\hline & & & Lateral & AP & Vertical & Sagittal & Coronal & \\
\hline \multirow{2}{*}{1} & $\mathrm{Lt}$ & \multirow{2}{*}{24.92} & 9.08 & 8.09 & 5.22 & 32.64 & 14.42 & $\mathrm{C}+1-2-, 1.5 \mathrm{~V}, 60 \mu \mathrm{sec}, 90 \mathrm{~Hz}$ \\
\hline & Rt & & 8.11 & 8.09 & 5.22 & 31.64 & 13.89 & $0-1-2+, 1.0 \mathrm{~V}, 60 \mu \mathrm{sec}, 90 \mathrm{~Hz}$ \\
\hline \multirow{2}{*}{2} & $\mathrm{Lt}$ & \multirow{2}{*}{27.56} & 10.23 & 4.2 & 5.3 & 22.48 & 8.52 & $\mathrm{C}+1-, 3.5 \mathrm{~V}, 90 \mu \mathrm{sec}, 140 \mathrm{~Hz}$ \\
\hline & Rt & & 10.7 & 2.71 & 4.55 & 32.74 & 6.29 & $\mathrm{C}+1-, 3.5 \mathrm{~V}, 90 \mu \mathrm{sec}, 140 \mathrm{~Hz}$ \\
\hline \multirow{2}{*}{3} & Lt & \multirow{2}{*}{23.44} & 10.25 & 6.03 & 3.58 & 40.82 & 16.04 & $\mathrm{C}+1-, 2.9 \mathrm{~V}, 60 \mu \mathrm{sec}, 140 \mathrm{~Hz}$ \\
\hline & Rt & & 12.92 & 5.03 & 2.33 & 12.46 & 9.61 & $\mathrm{C}+2-, 2.8 \mathrm{~V}, 60 \mu \mathrm{sec}, 140 \mathrm{~Hz}$ \\
\hline \multirow{2}{*}{4} & $\mathrm{Lt}$ & \multirow{2}{*}{24.44} & 10.29 & 7.1 & 3.41 & 16.53 & 8.86 & $\mathrm{C}+1-, 3.5 \mathrm{~V}, 60 \mu \mathrm{sec}, 140 \mathrm{~Hz}$ \\
\hline & Rt & & 10.14 & 7.1 & 3.41 & 18.84 & 8.26 & $\mathrm{C}+1-, 3.3 \mathrm{~V}, 60 \mu \mathrm{sec}, 140 \mathrm{~Hz}$ \\
\hline
\end{tabular}

* Anteroposterior and vertical coordinates were measured from the posterior commissure.

† The final settings were obtained at the 12-month follow-up in participants 1, 2, and 4 and at the 17-month follow-up in participant 3.

ments, from 0 to $4-5 \mathrm{~V}$. At each amplitude, the participant was queried on stimulation-related side effects and examined for changes in dystonic symptoms. Participants returned for postoperative programming and data collection visits bimonthly (except for participant 4 , who only had visits at 2,10 , and 12 months). DBS settings were only permitted to be changed every 8 weeks to allow for settings to reach steady state and fully allow for evaluation of effectiveness by caregivers and patients. Any improvement in dystonia during DBS programming was noted. This programming paradigm was based on historical data of the programming of GPi DBS in dystonia..$^{20}$ DBS stimulation settings at 12 months are listed in Table 1.

\section{Statistical Analysis}

Analysis of this phase 1 clinical trial was designed to assess the safety of continuous thalamic stimulation and to
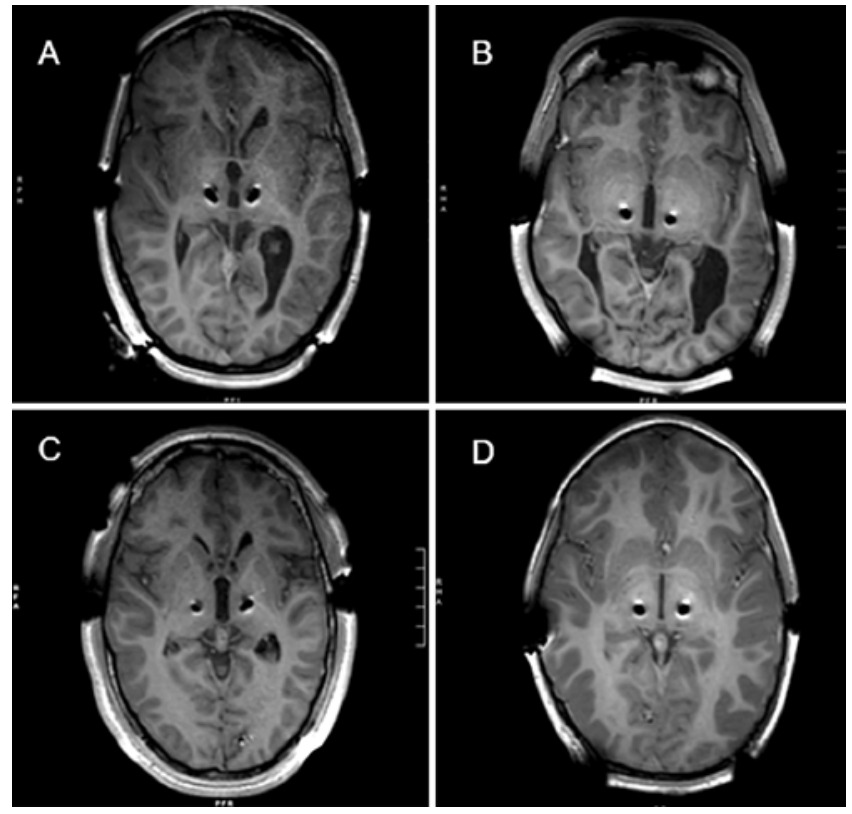

FIG. 2. Axial 3T T1-weighted MR images showing DBS lead tip artifacts for participants 1 (A), 2 (B), 3 (C), and 4 (D). estimate treatment effect size for the future design of larger-scale trials. The primary outcome of this analysis was variation in dystonia severity across the 12-month study period, as determined by differences in BFMDRS and BADS scores, while secondary outcomes included variation in spasticity, quality of life, and neuropsychological assessment. The nonparametric Wilcoxon matched-pairs signed-rank test was applied (2-tailed; $\alpha=0.05$ ) to study differences between baseline and 12-month outcomes.

\section{Adverse Events}

Prior to study inclusion, participants and their legal representatives were informed of the potential risks of undergoing DBS surgery in a less frequently studied brain target for this indication. During the trial, participants and representatives kept a log of stimulation-related adverse events and were interviewed in detail by the study team during every in-person evaluation for stimulation-related and other adverse events. Participants with implants and their representatives understood their unequivocal right to separate from the study at any time and the fact that the study team could terminate their participation for medical or safety reasons.

\section{Results}

\section{Participant Demographics}

Five prospective participants were screened, underwent comprehensive evaluations, and met inclusion criteria for enrollment. One declined further engagement prior to surgery, but the remaining 4 participants eventually underwent implantation and remained in the study for its duration. These 4 participants ( 2 males and 2 females) had a mean age (standard error) of 14.5 years (2 years) and a mean dystonia duration of 13 years (2.6 years). Demographic and clinical information for these 4 participants is listed in Table 2. Briefly, participant 1 developed spasticity and generalized dystonia following West Nile encephalitis at the age of 7 years. Participant 2 was born prematurely at 26 weeks after premature labor and low-lying placenta and displayed delay in all early psychomotor milestones and subsequently exhibited spastic dystonic quadriparesis. He had a known grade 4 
TABLE 2. Demographic and clinical characteristics

\begin{tabular}{|c|c|c|c|c|c|c|}
\hline $\begin{array}{l}\text { Participant } \\
\quad \text { No. }\end{array}$ & $\begin{array}{l}\text { Age at } \\
\text { Implantation } \\
\text { (yrs), Sex }\end{array}$ & $\begin{array}{c}\text { Race/ } \\
\text { Ethnicity }\end{array}$ & $\begin{array}{l}\text { Symptom } \\
\text { Duration } \\
\text { (yrs) }\end{array}$ & Diagnosis & GMFCS & $\begin{array}{l}\text { Procedure \& } \\
\text { Medication }\end{array}$ \\
\hline 1 & $14, \mathrm{~F}$ & $\begin{array}{l}\text { White/ } \\
\text { not } \\
\text { Hispanic }\end{array}$ & 8 & $\begin{array}{l}\text { West Nile } \\
\text { encephalitis at } \\
7 \text { yrs }\end{array}$ & IV & $\begin{array}{l}\text { Procedure: It hip intertrochanteric osteotomy w/ open reduction, } \\
\text { posterior spine arthrodesis T2 to pelvis } \\
\text { Pre-DBS medication: baclofen, diazepam, dantrolene, carbidopa/ } \\
\text { levodopa, botulinum toxin injections } \\
\text { Post-DBS medication: diazepam as needed, botulinum toxin injections } \\
\text { Previously failed medication: trihexyphenidyl }\end{array}$ \\
\hline 2 & $19, \mathrm{M}$ & $\begin{array}{l}\text { White/ } \\
\text { Hispanic }\end{array}$ & 19 & $\begin{array}{l}\text { Dystonic cerebral } \\
\text { palsy, premature } \\
\text { birth at } 26 \text { wks }\end{array}$ & IV & $\begin{array}{l}\text { Procedure: bilat hamstring tendon lengthening, dental restoration } \\
\text { Pre-DBS medication: baclofen } \\
\text { Post-DBS medication: baclofen } \\
\text { Previously failed medication: trihexyphenidyl, carbidopa/levodopa }\end{array}$ \\
\hline 3 & $16, M$ & $\begin{array}{l}\text { Other/ } \\
\text { not } \\
\text { Hispanic }\end{array}$ & 16 & $\begin{array}{l}\text { Dystonic cerebral } \\
\text { palsy, neonatal } \\
\text { encephalopathy }\end{array}$ & V & $\begin{array}{l}\text { Procedure: selective dorsal rhizotomy, bilat hip osteotomies, strabis- } \\
\text { mus repair, testicular detorsion } \\
\text { Pre-DBS medication: baclofen, botulinum toxin injections } \\
\text { Post-DBS medication: diazepam } \\
\text { Previously failed medication: none }\end{array}$ \\
\hline 4 & $9, F$ & $\begin{array}{l}\text { White/ } \\
\text { not } \\
\text { Hispanic }\end{array}$ & 9 & $\begin{array}{l}\text { Dystonic cerebral } \\
\text { palsy, neonatal } \\
\text { encephalopathy }\end{array}$ & II & $\begin{array}{l}\text { Procedure: none } \\
\text { Pre-DBS medication: none } \\
\text { Post-DBS medication: none } \\
\text { Previously failed medication: carbidopa/levodopa }\end{array}$ \\
\hline
\end{tabular}

GMFCS $=$ Gross Motor Function Classification System for cerebral palsy.

intraventricular hemorrhage with periventricular leukomalacia at birth. Participant 3 was born in cardiac arrest at week 37 via emergency cesarean section for cord prolapse and developed early spastic quadriparesis and generalized dystonia. Lastly, participant 4 was born at 39 weeks following presumed birth asphyxia and neonatal seizures and developed a mixture of dystonia and spasticity (Video 1).

VIDEO 1. Participant 4. Video recordings obtained preoperatively and 12 months postoperatively in a patient with dystonia and spasticity. Copyright Marta San Luciano. Published with permission.

Click here to view.

Details and T2-weighted preoperative MR images obtained in participants are shown in Fig. 3.

\section{Primary Outcomes}

The mean baseline motor BFMDRS and BADS scores were 75.4 (standard error 7.2) and 21.2 (1.4), respectively. The mean decrease in motor BFMDRS score following 12 months of continuous thalamic stimulation was 14.7 points (11.8 points) and ranged from a 0.5-point increase to a 25-point decrease. This represented an average improvement of $21.5 \%$. The total BADS score decreased by an average of 0.25 points ( 3.2 points) and ranged from a 2-point increase to a 3-point decrease, a $1.6 \%$ improvement. These differences were not statistically significant after application of the nonparametric Wilcoxon matchedpairs signed-rank test $(\mathrm{p}=0.14$ for the motor BFMDRS score and $\mathrm{p}=0.70$ for the BADS score). In the blinded video review, there was an average decrease in the motor BFMDRS score of 0.33 points ( 3 points; $p=0.99$ ) and an increase of 2 points (1.7 points; $\mathrm{p}=0.31)$ in the BADS score. Primary efficacy outcomes are displayed in Table 3.

\section{Secondary Outcomes}

All participants had upper- and lower-limb spasticity at baseline (average Modified Ashworth Scale scores for upper and lower limbs of 1.9 and 1.7, respectively; Table 4). At 12 months, there was a nonsignificant average increase in spasticity in both the upper and lower limbs (0.29- and 0.26-point increases in upper and lower limbs, respectively; $\mathrm{p}=0.57$ in both cases). The participants, their caregivers, and clinicians involved in the study did not consider the increase in spasticity demonstrated in the scales as clinically relevant. Instead, all caregivers reported that the home physical therapists reported reduced tone and increased passive mobility compared with prior to surgery.

The BFMDRS disability subscale showed wide variability, but the score improved by an average of $15.7 \%$ compared with baseline (range 9.1\% worsening to $53.8 \%$ improvement, $\mathrm{p}=0.23$ ), and the PedsQL score improved an average of $27 \%$ (range $0 \%$ to $54.8 \%$ improvement, $\mathrm{p}$ $=0.09$ ). The modified UPDRS-II score was available at 12 months for only 2 participants and showed improvements of $23.5 \%$ for one participant and $15 \%$ in the other (Table 5). Follow-up neuropsychological testing at 12-25 months postoperatively did not show clinically meaningful worsening in any domain, and the findings remained stable overall (Supplementary Table).

\section{Subjective and Functional Outcomes}

Despite nonsignificant changes in the rating scores, improved functionality and the ability to perform daily tasks were reported in all but one patient, and participants reported qualitative improvements. As an example, par- 

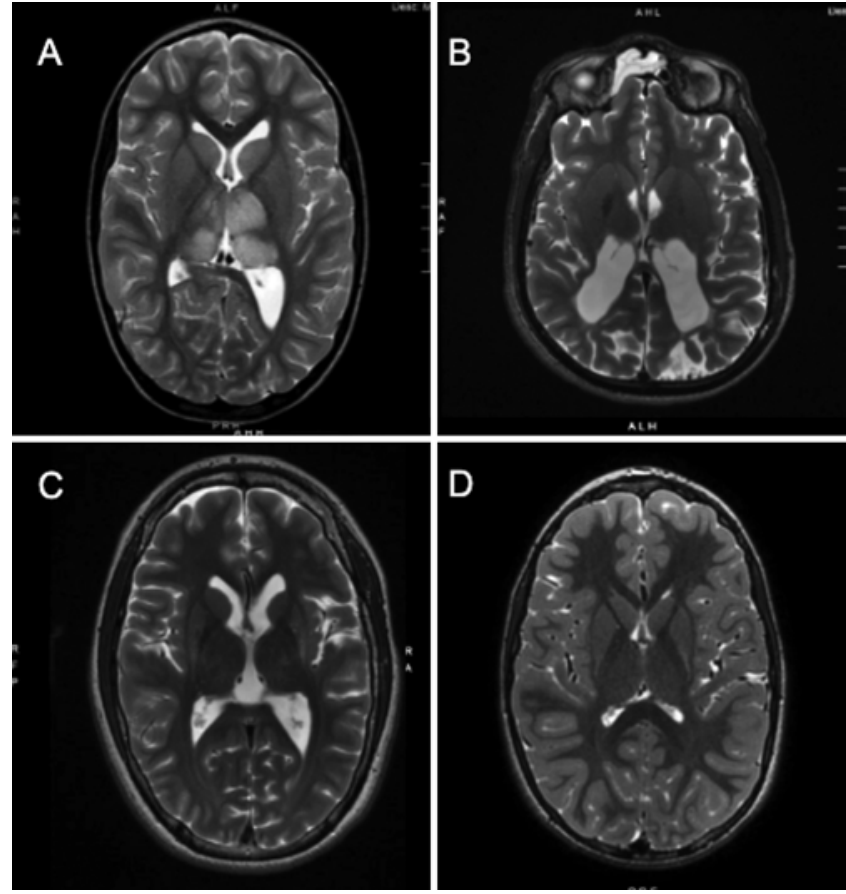

FIG. 3. A: Participant 1. Preoperative axial T2-weighted MR image showing vermis and pontine atrophy, abnormal cerebellar signal, and diffusely diminished supratentorial white matter with thinning of the corpus callosum. B: Participant 2. Preoperative axial T2-weighted MR image obtained at the age of 19 years, showing posterior predominant cerebral as well as bilateral thalamic volume loss and posterior corpus callosum atrophy. C: Participant 3. Preoperative axial brain MR image showing global cerebral, bilateral thalamic, and left more than right putaminal volume loss, as well as Wallerian degeneration of bilateral internal capsules and cerebral peduncles. D: Participant 4. Preoperative axial T2-weighted MR image showing very mild T2 hyperintensities in bilateral pallidum, posterior putamen, ventrolateral thalami, and perirolandic regions, representing sequelae of prior injury.

ticipant 4, who experienced frequent falls prior to surgery, displayed no measurable changes in ambulatory status but exhibited a significant reduction in the frequency of her falls and even gained the ability to run. Because of these improvements, she had removal of restrictions on the playground, and she no longer required a helmet to keep her safe (Video 1). Participant 2 no longer required restraint of his upper limbs to avoid self-harm from dystonic movements. Three participants exhibited improvements in sleep due to increased ability to turn over in bed, and caregivers reported improvement in transfers and gain of some independence with toileting.

Anecdotally, during the postoperative neuropsychological evaluations, there was reported improvement in assessments of depression, anxiety and irritability, and psychotic symptoms, and improved sleep in 3 participants (Supplementary Table).

\section{Safety Outcomes}

All participants tolerated the procedure well, and there were no safety concerns or serious adverse events. Some degree of anticipated mild stimulation-related temporary side effects was experienced by all participants, particu- larly during the initial programming monopolar review, where the neurostimulator was interrogated to determine the maximum tolerable voltage. Each of these side effects, which included paresthesia of the tongue and limbs, dysarthria and mouth pulling, transient increase in bothersome movements, and feelings of discomfort, were resolved by lowering the voltage. Additional unanticipated adverse effects included postoperative diarrhea, which was likely related to the precautionary antibiotic course. One participant developed new speech difficulties, decreased energy, and an unwillingness to assist with rolling over following stimulation changes at the 10-month visit (perceived by family as apathy). These side effects were initially attributed to a viral infection but resolved after lowering the voltage slightly.

\section{Discussion}

This open-label phase 1 trial evaluating the preliminary efficacy and safety of bilateral Vop/Vim thalamic DBS for severe acquired dystonia in 4 children and young adults showed feasibility, adequate tolerability and safety, and some encouraging preliminary results.

Thalamic stimulation presented no irreversible complications. Beyond the expected stimulation-induced side effects present during monopolar review and 1 episode of potential transient stimulation-induced apathy, there were no long-term or permanent side effects or complications. Notably, there were also no infections, which is the most common complication in this study population. There is uncertainty in the lead targeting with respect to the Vim/ Vop boundary in the anteroposterior direction, given the inability to distinguish these nuclei anatomically at 3T. While microelectrode recording could have been used in principle to map the boundary between the motor and sensory thalamus, this method is impractical in asleep patients undergoing implantation with MRI guidance. Postoperative imaging confirmed that the leads were implanted within $1 \mathrm{~mm}$ (radial error) of the intended target.

Primary and secondary outcomes revealed highly variable effect sizes with unblinded severity ratings showing 21.5\% improvement in BFMDRS at 12 months, which are similar to previously reported outcomes of pallidal stimulation. $^{21,22}$ Unsurprisingly, the participant with the shortest dystonia duration, who was also the youngest, saw the greatest improvement. She also was the most functional at baseline and had the least structural damage seen on MRI, both of which have been shown to be likely predictive factors of success rates for pallidal stimulation. ${ }^{21,23,24}$

Acquired dystonia, especially secondary to perinatal injuries (commonly clinically referred to as cerebral palsy), represents one of the most common causes of dystonia in children and young adults. The overall reported prevalence of cerebral palsy in children aged 3-10 years is 2.4 per 1000 children; movement disorders, including dystonia, athetosis, and chorea, are common manifestations, ${ }^{3,12}$ affecting up to $15 \%$ of patients. ${ }^{25}$ The surgical treatment of severe acquired dystonia in cerebral palsy remains an important challenge.

A 2013 metanalysis of pallidal DBS in acquired dystonia that included 19 unblinded studies showed overall im- 
TABLE 3. Primary efficacy outcomes

\begin{tabular}{|c|c|c|c|c|c|c|c|c|c|c|}
\hline \multirow{2}{*}{$\begin{array}{c}\text { Participant } \\
\text { No. }\end{array}$} & \multicolumn{5}{|c|}{ BFMDRS (unblinded) } & \multicolumn{5}{|c|}{ BFMDRS (blinded) } \\
\hline & $B L$ & 6 Mos & 12 Mos & $\Delta$ & $\% \Delta$ & $\mathrm{BL}$ & 6 Mos & 12 Mos & $\Delta$ & $\% \Delta$ \\
\hline 1 & 77.5 & 60 & 78 & 0.5 & 0.6 & 60.5 & 80 & 62.5 & 2 & 3.3 \\
\hline 2 & 92 & 77 & 69 & -23 & -25 & 66 & 69.5 & 70 & 4 & 6.1 \\
\hline $3^{*}$ & 79 & 55.5 & 67.5 & -11.5 & -14.6 & 63 & 75.5 & 80.5 & 17.5 & 27.8 \\
\hline 4 & 53 & & 28 & -25 & -47.2 & 40 & & 33 & -7 & -17.5 \\
\hline \multirow{2}{*}{$\begin{array}{l}\text { Participant } \\
\text { No. }\end{array}$} & \multicolumn{5}{|c|}{ BADS (unblinded) } & \multicolumn{5}{|c|}{ BADS (blinded) } \\
\hline & $B L$ & 6 Mos & 12 Mos & $\Delta$ & $\% \Delta$ & $\mathrm{BL}$ & 6 Mos & 12 Mos & $\Delta$ & $\% \Delta$ \\
\hline 1 & 21 & 20 & 23 & 2 & 9.5 & 24 & 23 & 22 & -2 & -8.3 \\
\hline 2 & 25 & 23 & 22 & -3 & -12 & 21 & 23 & 25 & 4 & 19 \\
\hline $3^{*}$ & 22 & 19 & 25 & 3 & 13.6 & 21 & 27 & 26 & 5 & 23.8 \\
\hline 4 & 17 & & 14 & -3 & -17.6 & 13 & & 17 & 4 & 30.8 \\
\hline
\end{tabular}

${ }^{*}$ For participant 3 , results from the 10 -month evaluation were used because the 12-month blinded outcomes were unavailable.

provements of $23.6 \%$ in BFMDRS motor scores and $9.2 \%$ in BFMDRS disability scores, ${ }^{6}$ with greater improvement seen among younger individuals. More recent studies confirm highly variable and often-disappointing gross motor responses in other etiologies apart from isolated dystonia, ${ }^{26,27}$ including acquired, heredodegenerative, and idiopathic dystonia, highlighting the need for alternative brain targets.

There is growing recognition that brain structures outside of the basal ganglia are involved in dystonia, and dystonia is now generally understood as a network disorder. ${ }^{13,28}$ Dysfunction of the cerebellum and related thalamocortical circuits can lead to dystonia, likely through dysfunctional interactions with the basal ganglia circuitry, and neuromodulation of such cerebellothalamic pathways may improve dystonic symptoms..$^{13,29}$ In isolated dystonia, for example, targeting of the Vim is now considered to be an equivalent, or even superior, approach to targeting the GPi for the management of dystonic tremor, as well as for cases in which tremor is the patient's most disabling symptom..$^{30,31}$ In acquired dystonia, which is considered

\section{TABLE 4. Modified Ashworth Scale spasticity ratings}

\begin{tabular}{|c|c|c|c|c|}
\hline \multirow{2}{*}{$\begin{array}{c}\text { Participant } \\
\text { No. }\end{array}$} & \multicolumn{4}{|c|}{ Upper-Extremity Averages } \\
\hline & $\mathrm{BL}$ & 6 Mos & $12 \mathrm{Mos}$ & $\% \Delta$ \\
\hline 1 & 1.57 & 0.63 & 2.83 & 80.3 \\
\hline 2 & 2.25 & 2.5 & 2 & -12.5 \\
\hline 3 & 3 & 1.33 & 3.13 & 4.3 \\
\hline 4 & 0.75 & & 0.75 & 0 \\
\hline \multirow{2}{*}{$\begin{array}{c}\text { Participant } \\
\text { No. }\end{array}$} & \multicolumn{4}{|c|}{ Lower-Extremity Averages } \\
\hline & $\mathrm{BL}$ & $6 \mathrm{Mos}$ & $12 \mathrm{Mos}$ & $\% \Delta$ \\
\hline 1 & 1.38 & 0.63 & 2.67 & 93.5 \\
\hline 2 & 2.13 & 2 & 1.5 & -29.6 \\
\hline 3 & 2.5 & 0.88 & 2.5 & 0 \\
\hline 4 & 0.63 & & 1 & 58.7 \\
\hline
\end{tabular}

etiologically multifactorial and in which thalamocerebellar pathways are frequently affected, ${ }^{32}$ both cerebellar ${ }^{33}$ and thalamic targets have been explored. Thalamic stimulation has been explored for the treatment of acquired dystonia and choreoathetosis in a handful of small heterogeneous studies but mostly in combination with pallidal stimulation. Wolf et al. reported on concurrent pallidal and Vim stimulation among 3 adult patients with cerebral palsy, with results equivalent to those of pallidal stimu-

TABLE 5. Quality-of-life outcomes

\begin{tabular}{|c|c|c|c|c|c|}
\hline \multirow{2}{*}{$\begin{array}{l}\text { Participant } \\
\text { No. }\end{array}$} & \multicolumn{5}{|c|}{ BFMDRS Disability Total } \\
\hline & $B L$ & $6 \mathrm{Mos}$ & 12 Mos & $\Delta$ & $\% \Delta$ \\
\hline 1 & 28 & 26 & 24 & -4 & -14.3 \\
\hline 2 & 25 & 24 & 24 & -1 & -4 \\
\hline 3 & 22 & 21 & 24 & 2 & 9.1 \\
\hline 4 & 13 & & 6 & -7 & -53.8 \\
\hline \multirow{2}{*}{$\begin{array}{l}\text { Participant } \\
\text { No. }\end{array}$} & \multicolumn{5}{|c|}{ Modified UPDRS-II Total } \\
\hline & $B L$ & 6 Mos & 12 Mos & $\Delta$ & $\% \Delta$ \\
\hline 1 & 38 & 36 & & & \\
\hline 2 & 35 & 33 & & & \\
\hline 3 & 34 & 29 & 26 & -8 & -23.5 \\
\hline 4 & 20 & & 17 & -3 & -15 \\
\hline \multirow{2}{*}{$\begin{array}{l}\text { Participant } \\
\text { No. }\end{array}$} & \multicolumn{5}{|c|}{ PedsQL Total } \\
\hline & $\mathrm{BL}$ & 6 Mos & 12 Mos & $\Delta$ & $\% \Delta$ \\
\hline 1 & 49 & 48 & 44 & -5 & -10.2 \\
\hline 2 & 32 & 41 & 32 & 0 & 0 \\
\hline 3 & 42 & 37 & 19 & -23 & -54.8 \\
\hline 4 & 44 & & 25 & -19 & -43.2 \\
\hline
\end{tabular}

The BFMDRS disability and modified UPDRS-II scales are scored such that higher numbers indicate greater disease severity and lower numbers indicate lesser disease severity. Similarly, the PedsQL scale is scored such that higher numbers indicate decreased health-related quality of life and lower numbers indicate higher health-related quality of life. 
lation alone for all but one patient. ${ }^{12}$ In a separate study, the addition of Vop leads to pallidal DBS was effective in addressing complex movement disorders in 2 patients with neuroacanthocytosis, ${ }^{34}$ and stimulation of the Vop itself improved severe trunk spasms in another patient with neuroacanthocytosis. ${ }^{35}$ The addition of bilateral ventralis oralis anterior electrodes to pallidal DBS also brought marked improvements in a single participant with severe postanoxic dystonia and bilateral basal ganglia necrosis. ${ }^{36}$

Specific targeting of the Vop and Vim according to electrophysiological perioperative data using stereo-EEG depth electrodes in children with acquired and combined dystonia has led to improved outcomes in a novel approach to DBS target selection. ${ }^{11}$ Stimulation of the combined Vop/Vim area, which has the potential to affect both pallidal and cerebellar inputs and provide a more efficient therapeutic option for this population, has not been previously studied in a rigorous manner.

Our study found improvements to quality of life that were not captured by validated rating scales. These included an increased ability to turn over in bed, which, in turn, reportedly improved sleep quality; improvement in transfers and toileting; greater weight-bearing ability during walking; decreased reliance on physical restraints; and fewer falls during ambulation. Additionally, there was reported improvement in psychiatric symptoms (depression, anxiety, irritability, and psychosis) in 3 of the 4 participants, with the most functional participant showing the greatest benefits. Despite these reported improvements in quality of life and mood, unblinded BADS scores and blinded video BFMDRS and BADS scores did not reflect the same degree of improvement. This discrepancy has been noted in many other studies of DBS in cerebral palsy and other acquired dystonias. ${ }^{23,37,38}$ Current gold-standard rating scales relied on by movement disorder neurologists to assess severity and disability do not adequately capture many of the symptoms that may improve with DBS. As a result, symptomatic improvements such as those witnessed in our study may only be detected with a careful review of systems. ${ }^{23}$ The current dystonia rating scales also fail to adequately measure individual limb components and cannot account for spasticity and joint contractures, common features of mixed movement disorders in acquired dystonia. ${ }^{3,23}$ Hence, the differences found between our blinded and unblinded ratings are likely due to a combination of lower interrater reliability and sensitivity in children, the difference between observation times during in-person evaluations and the shorter videotaped clips, and biased outcome ascertainment due to a lack of blinding. These findings highlight the importance of performing adequate blinded evaluations in future trials and, as previously suggested, ${ }^{39,40}$ the need to develop rating scales more suitable to acquired dystonia. Application of wearable technology, perhaps along with video analysis to quantify objective changes in complex movements, may provide a solution to this problem. Systems such as these, which would more easily quantify subtle symptoms, may prove to be a superior alternative to simply relying on adaptations of existing scales for movement disorders developed specifically for isolated dystonia and adult-onset movement disorders. Until such systems can be integrated, clinical studies of pediatric DBS should continue to rely on best-available measures, including open-ended symptom reviews and quality-of-life measures, to fully capture the benefits and pitfalls of DBS.

There is an unmet need for methods to reliably determine intellectual capacity and cognitive ability in patients with severe motor impairments. Attempts to circumvent this necessity by individualizing neuropsychological testing still brought significant challenges. We attribute some of these challenges to oculomotor apraxia, which limited the utility of nonverbal cognitive assessments. There were also other difficulties in discerning which answer the participant intended to give due to limitations from dystonia, physical and mental fatigue, and differential life experiences relative to those typically experienced by children without significant motor impairment.

Our study represents one of only a few prospective studies of DBS involving children. Significant strengths of our study include the well-characterized preoperative and postoperative profiles of our cohort, use of validated outcome assessments, and inclusion of blinded video ratings. Limitations, however, include the small number of participants who underwent implantation and the etiological heterogeneity of those patients successfully recruited, despite the commonality of all 4 participants having sustained structural brain damage. However, even in cases in which acquired dystonia could be limited to one cause, for example, hypoxic-ischemic injury at birth, the distribution of brain damage will always vary between participants, making a homogeneous participant population nearly impossible.

\section{Conclusions}

Bilateral Vop/Vim thalamic neuromodulation by DBS is feasible, well tolerated, and safe for severe acquired dystonia. While the measurement tools used in this study were unable to truly capture the potential benefits from DBS, this study presents a feasible framework for carrying out clinical trials of neuromodulation in pediatric patients with extreme physical disabilities and communication difficulties. Most pediatric DBS studies rely on either retrospective analyses or summaries of case reports, and our study provides additional evidence of the feasibility of testing clinical trial designs, as well as of collecting data in this exceptionally challenging group of patients. Given the large response variability between patients, novel N-of-1 experimental designs with multiple crossover doubleblinded replications of effective and ineffective stimulation per participant, rather than classic parallel group comparisons, may be better suited to determine DBS efficacy in acquired dystonia.

\section{Acknowledgments}

This study was supported by the Smart Family Foundation (to M.S.L.), NIH/NINDS K23NS0099441-O1A (to M.S.L.), and NIH/NINDS K12NS001692 (to A.R.V.). We thank the participants and their caregivers for their participation.

\section{References}

1. Albanese A, Bhatia K, Bressman SB, et al. Phenomenology and classification of dystonia: a consensus update. Mov Disord. 2013;28(7):863-873. 
2. Mink JW. Special concerns in defining, studying, and treating dystonia in children. Mov Disord. 2013;28(7):921-925.

3. Koman LA, Smith BP, Shilt JS. Cerebral palsy. Lancet. 2004; 363(9421):1619-1631.

4. Kupsch A, Benecke R, Müller J, et al. Pallidal deep-brain stimulation in primary generalized or segmental dystonia. $N$ Engl J Med. 2006;355(19):1978-1990.

5. Brüggemann N, Kühn A, Schneider SA, et al. Short- and long-term outcome of chronic pallidal neurostimulation in monogenic isolated dystonia. Neurology. 2015;84(9):895-903.

6. Koy A, Hellmich M, Pauls KA, et al. Effects of deep brain stimulation in dyskinetic cerebral palsy: a meta-analysis. Mov Disord. 2013;28(5):647-654.

7. Burchiel KJ. Thalamotomy for movement disorders. Neurosurg Clin N Am. 1995;6(1):55-71.

8. Cardoso F, Jankovic J, Grossman RG, Hamilton WJ. Outcome after stereotactic thalamotomy for dystonia and hemiballismus. Neurosurgery. 1995;36(3):501-508.

9. Broggi G, Angelini L, Giorgi C. Neurological and psychological side effects after stereotactic thalamotomy in patients with cerebral palsy. Neurosurgery. 1980;7(2):127-134.

10. Patel A, Deeb W, Okun MS. Deep brain stimulation management of essential tremor with dystonic features. Tremor Other Hyperkinet Mov (N Y). 2018;8:557.

11. Sanger TD, Liker M, Arguelles E, et al. Pediatric deep brain stimulation using awake recording and stimulation for target selection in an inpatient neuromodulation monitoring unit. Brain Sci. 2018;8(7):135.

12. Wolf ME, Blahak C, Saryyeva A, et al. Deep brain stimulation for dystonia-choreoathetosis in cerebral palsy: pallidal versus thalamic stimulation. Parkinsonism Relat Disord. 2019;63:209-212.

13. Jinnah HA, Neychev V, Hess EJ. The anatomical basis for dystonia: the motor network model. Tremor Other Hyperkinet $\operatorname{Mov}(N Y)$. 2017;7:506.

14. Marshall RD, Collins A, Escolar ML, et al. A scale to assess activities of daily living in pantothenate kinase-associated neurodegeneration. Mov Disord Clin Pract (Hoboken). 2019; 6(2):139-149.

15. Kaufman AS, Kaufman NL. Kaufman Brief Intelligence Test. 2nd ed. Pearson; 2004.

16. Kamphaus RW, Reynolds CR. Behavior Assessment System for Children-Third Edition (BASC-3): Behavioral and Emotional Screening System (BEES). Pearson; 2015.

17. American Psychiatric Association. Diagnostic and Statistical Manual of Mental Disorders. 5th ed. APA; 2013.

18. Starr PA, Markun LC, Larson PS, et al. Interventional MRIguided deep brain stimulation in pediatric dystonia: first experience with the ClearPoint system. J Neurosurg Pediatr. 2014;14(4):400-408.

19. Olaya JE, Christian E, Ferman D, et al. Deep brain stimulation in children and young adults with secondary dystonia: the Children's Hospital Los Angeles experience. Neurosurg Focus. 2013;35(5):E7.

20. Kupsch A, Tagliati M, Vidailhet M, et al. Early postoperative management of DBS in dystonia: programming, response to stimulation, adverse events, medication changes, evaluations, and troubleshooting. Mov Disord. 2011;26(suppl 1):S37-S53.

21. Vidailhet M, Yelnik J, Lagrange C, et al. Bilateral pallidal deep brain stimulation for the treatment of patients with dystonia-choreoathetosis cerebral palsy: a prospective pilot study. Lancet Neurol. 2009;8(8):709-717.

22. Krauss JK, Loher TJ, Weigel R, Capelle HH, Weber S, Burgunder J-M. Chronic stimulation of the globus pallidus internus for treatment of non-DYT1 generalized dystonia and choreoathetosis: 2-year follow up. J Neurosurg. 2003;98(4):785.

23. Marks WA, Honeycutt J, Acosta F Jr, et al. Dystonia due to cerebral palsy responds to deep brain stimulation of the globus pallidus internus. Mov Disord. 2011;26(9):1748-1751.
24. Lumsden DE, Kaminska M, Gimeno H, et al. Proportion of life lived with dystonia inversely correlates with response to pallidal deep brain stimulation in both primary and secondary childhood dystonia. Dev Med Child Neurol. 2013;55(6): 567-574.

25. Treves T, Korczyn AD. Progressive dystonia and paraparesis in cerebral palsy. Eur Neurol. 1986;25(2):148-153.

26. Tustin K, Elze MC, Lumsden DE, et al. Gross motor function outcomes following deep brain stimulation for childhoodonset dystonia: a descriptive report. Eur J Paediatr Neurol. 2019;23(3):473-483.

27. Koy A, Weinsheimer M, Pauls KAM, et al. German registry of paediatric deep brain stimulation in patients with childhood-onset dystonia (GEPESTIM). Eur J Paediatr Neurol. 2017;21(1):136-146.

28. Prudente CN, Hess EJ, Jinnah HA. Dystonia as a network disorder: what is the role of the cerebellum? Neuroscience. 2014;260:23-35.

29. Sanger TD. A computational model of deep-brain stimulation for acquired dystonia in children. Front Comput Neurosci. 2018;12:77.

30. Hedera P, Phibbs FT, Dolhun R, et al. Surgical targets for dystonic tremor: considerations between the globus pallidus and ventral intermediate thalamic nucleus. Parkinsonism Relat Disord. 2013;19(7):684-686.

31. Fasano A, Bove F, Lang AE. The treatment of dystonic tremor: a systematic review. J Neurol Neurosurg Psychiatry. 2014; 85(7):759-769.

32. Qin Y, Li Y, Sun B, et al. Functional connectivity alterations in children with spastic and dyskinetic cerebral palsy. Neural Plast. 2018;2018:7058953.

33. Brown EG, Bledsoe IO, Luthra NS, et al. Cerebellar deep brain stimulation for acquired hemidystonia. Mov Disord Clin Pract (Hoboken). 2020;7(2):188-193.

34. Nakano N, Miyauchi M, Nakanishi K, et al. Successful combination of pallidal and thalamic stimulation for intractable involuntary movements in patients with neuroacanthocytosis. World Neurosurg. 2015;84(4):1177.e1-1177.e7.

35. Burbaud P, Rougier A, Ferrer X, et al. Improvement of severe trunk spasms by bilateral high-frequency stimulation of the motor thalamus in a patient with chorea-acanthocytosis. Mov Disord. 2002;17(1):204-207.

36. Ghika J, Villemure JG, Miklossy J, et al. Postanoxic generalized dystonia improved by bilateral Voa thalamic deep brain stimulation. Neurology. 2002;58(2):311-313.

37. Kim AR, Chang JW, Chang WS, et al. Two-year outcomes of deep brain stimulation in adults with cerebral palsy. Ann Rehabil Med. 2014;38(2):209-217.

38. Lin JP, Lumsden DE, Gimeno H, Kaminska M. The impact and prognosis for dystonia in childhood including dystonic cerebral palsy: a clinical and demographic tertiary cohort study. J Neurol Neurosurg Psychiatry. 2014;85(11):12391244.

39. Elze MC, Gimeno H, Tustin K, et al. Burke-Fahn-Marsden dystonia severity, Gross Motor, Manual Ability, and Communication Function Classification scales in childhood hyperkinetic movement disorders including cerebral palsy: a 'Rosetta Stone' study. Dev Med Child Neurol. 2016;58(2):145-153.

40. Gimeno H, Tustin K, Selway R, Lin JP. Beyond the BurkeFahn-Marsden Dystonia Rating Scale: deep brain stimulation in childhood secondary dystonia. Eur J Paediatr Neurol. 2012;16(5):501-508.

\section{Disclosures}

Dr. San Luiciano: consultant for Boston Scientific. Dr. Bledsoe: consultant for Boston Scientific. Dr. Starr: support of non-studyrelated clinical or research effort from Medtronic and Boston Scientific. Dr. Ostrem: consultant for Abbot and support of 
non-study-related clinical or research effort from Medtronic and Boston Scienfic.

\section{Author Contributions}

Conception and design: San Luciano, Robichaux-Viehoever, Starr, Ostrem. Acquisition of data: San Luciano, Robichaux-Viehoever, Dodenhoff, Gittings, Racine, Bledsoe, Watson Pereira, Wang, Starr, Ostrem. Analysis and interpretation of data: San Luciano, Robichaux-Viehoever, Racine, Bledsoe, Starr, Ostrem. Drafting the article: San Luciano, Robichaux-Viehoever. Critically revising the article: Robichaux-Viehoever, Dodenhoff, Gittings, Viser, Racine, Bledsoe, Watson Pereira, Wang, Starr, Ostrem. Reviewed submitted version of manuscript: all authors. Approved the final version of the manuscript on behalf of all authors: San Luciano. Statistical analysis: San Luciano. Administrative/technical/material support: Dodenhoff, Gittings, Viser, Wang. Study supervision: San Luciano, Robichaux-Viehoever, Wang, Starr, Ostrem.

\section{Supplemental Information \\ Videos}

Video 1. https://vimeo.com/445198460.

\section{Online-Only Content}

Supplemental material is available with the online version of the article.

Supplementary Table. https://thejns.org/doi/suppl/10.3171/ 2020.7.PEDS20348.

\section{Correspondence}

Marta San Luciano: Weill Institute for Neurosciences, University of California, San Francisco, CA. marta.sanlucianopalenzuela@ ucsf.edu. 